Article

\title{
Limitation of Cytokinin Export to the Shoots by Nucleoside Transporter ENT3 and Its Linkage with Root Elongation in Arabidopsis
}

\author{
Alla Korobova ${ }^{1}$, Bulat Kuluev ${ }^{2,3}$, Torsten Möhlmann ${ }^{4}\left[\right.$, Dmitriy Veselov ${ }^{1}$ and Guzel Kudoyarova ${ }^{1,3, *}$ \\ 1 Laboratory of Plant Physiology, Ufa Institute of Biology, Ufa Federal Research Centre, \\ RAS, 450054 Ufa, Russia; muksin@mail.ru (A.K.); veselov@anrb.ru (D.V.) \\ 2 Institute of Biochemistry and Genetics, Ufa Federal Research Centre, RAS, 450054 Ufa, Russia; kuluev@bk.ru \\ 3 Biological Department, Bashkir State University, 450076 Ufa, Russia \\ 4 Department of Biology, University of Kaiserslautern, 67663 Kaiserslautern, Germany; \\ moehlmann@biologie.uni-kl.de \\ * Correspondence: guzel@anrb.ru; Tel.: +7-9191427994
}

Citation: Korobova, A.; Kuluev, B.;

Möhlmann, T.; Veselov, D.;

Kudoyarova, G. Limitation of Cytokinin Export to the Shoots by Nucleoside Transporter ENT3 and Its Linkage with Root Elongation in Arabidopsis. Cells 2021, 10, 350. https://doi.org/10.3390/cells10020350

Academic Editor: Francesco Carimi

Received: 18 December 2020

Accepted: 6 February 2021

Published: 8 February 2021

Publisher's Note: MDPI stays neutral with regard to jurisdictional claims in published maps and institutional affiliations.

Copyright: (c) 2021 by the authors. Licensee MDPI, Basel, Switzerland. This article is an open access article distributed under the terms and conditions of the Creative Commons Attribution (CC BY) license (https:// creativecommons.org/licenses/by/ $4.0 /)$.

\begin{abstract}
The trans-membrane carrier AtENT3 is known to transport externally supplied cytokinin ribosides and thus promote uptake by cells. However, its role in distributing either exogenous or endogenous cytokinins within the intact plant has not hitherto been reported. To test this, we used ent3-1 mutant Arabidopsis seedlings in which the gene is not expressed due to a T-DNA insertion, and examined the effect on the concentration and distribution of either endogenous cytokinins or exogenous trans-zeatin riboside applied to the roots. In the mutant, accumulation of endogenous cytokinins in the roots was reduced and capacity to deliver externally supplied trans-zeatin riboside to the shoots was increased suggesting involvement of equilibrative nucleoside (ENT) transporter in the control of cytokinin distribution in the plants. Roots of ent3-1 were longer in the mutant in association with their lower cytokinin concentration. We concluded that the ENT3 transporter participates in partitioning endogenous cytokinins between the apoplast and the symplast by facilitating their uptake by root cells thereby limiting cytokinin export to the shoots through the xylem. Dilution of the mineral nutrient solution lowered endogenous cytokinin concentration in the roots of both wild type (WT) and ent3-1 plants accompanied by promotion of root elongation. Nevertheless, cytokinin content was lower, while roots were longer in the ent3-1 mutant than in the WT under either normal or deficient mineral nutrition suggesting a significant role of ENT3 transporter in the control of cytokinin level in the roots and the rate of their elongation.
\end{abstract}

Keywords: Arabidopsis thaliana; ENT3; exogenous cytokinin riboside; endogenous cytokinins; membrane transporters; root elongation

\section{Introduction}

Regulation of cytokinin concentration and distribution in plants is important for the control of their adaptation to environmental changes. A decline in the content of these hormones in shoots has been frequently detected under water deficiency in tobacco [1] and tomato [2], as well as under deficiency of mineral nutrients in Arabidopsis [3], wheat [4], barley [5], and rice [6]. Cytokinins are known to exert opposite effects on shoots and roots, promoting the shoot growth, while inhibiting the root growth [7]. Consequently, a reduction in cytokinin levels detected under deficiency of mineral nutrients or water [8] should inhibit shoot growth, while at the same time promoting root growth. Cytokinins have been shown to inhibit root elongation [9] and accumulation of root biomass [10] and consequently a decline in root cytokinin concentration is expected to activate root growth [6]. There are various mechanisms regulating cytokinin concentration under the changing environment. Thus, activation of cytokinin oxidases, resulting in inactivation 
of these hormones, served as a cause of the decline in cytokinin content in wheat plants exposed to diluted nutrient solution [4]. Addition of nitrates to the nutrient medium increased cytokinin level in plants brought about by up-regulation of IPT genes responsible for the synthesis of cytokinins in roots of maize [11] and leaves of Arabidopsis [12].

An important role for the regulation of cytokinins levels in plants is attributed to their transport from roots to shoots [13]. Although expression of IPT genes was detected not only in the roots but also in the shoots [12], the decline in capacity of roots for cytokinin export resulted in reduced accumulation of cytokinins in the shoots [14], confirming the importance of roots for the supply of shoots with these hormones. In these experiments, disturbance of cytokinin distribution between roots and shoots was due to mutation of the gene coding for ABCG14, an ABC-transporter enabling the export/efflux of these hormones from root cells, which contributes to cytokinin loading into the xylem. The shootward transport of root-derived cytokinins was significantly suppressed in rice plants with mutation in $\mathrm{ABC}$ transporter OsABCG18 [15].

Alongside $\mathrm{ABC}$ carriers, transport of cytokinins across membranes may be performed by purine permeases (PUPs) specialized in transport of nitrogenous bases [16] and equilibrative nucleoside (ENT)-transporters capable of transferring their ribosides [17]. Unlike ABC proteins, PUPs and ENT transporters are believed to support cytokinin uptake by the cells and not outflow of the hormones from the cells [18]. Recently attention has been drawn to the role of PUP-carriers for the transport of cytokinins $[19,20]$, but there is far less information concerning transporters of cytokinin ribosides. Although free cytokinin bases are known to be the active form of cytokinins, ribosylated cytokinins are the dominant form of these hormones present in the xylem sap and transported from roots to shoots [21]. Therefore, ENT transporters specialized in the trans-membrane transfer of ribosylated nitrogenous bases are likely to be involved in the transport of cytokinins from roots to shoots. However this possibility has not been studied so far. It is of interest that corresponding reporter constructs co-localized expression of the genes coding for IPT [12], ENT [22], and nucleoside hydrolase, NSH1 [23] in the root vasculature. The results suggest coordinated control of cytokinins synthesis, metabolism, and transport brought about by co-localization of corresponding participants of this network.

In the present work we have focused on ENT3, as this carrier represents the main nucleoside importer at the plasma-membrane in Arabidopsis. Studies of the uptake of radiolabeled nucleosides into yeast (Saccharomyces cerevisiae) cells and electrophysiological measurements on Xenopus oocytes expressing ENT3 clearly showed involvement of ENT3 in high affinity nucleoside/proton symport [24,25]. In addition, reduced uptake of nucleosides by hypocotyl explants of an ENT3 T-DNA insertion mutant (ent3-1) was observed in seedlings, leaf discs, and roots from hydroponically grown plants [25]. Most importantly, the efficiency of uptake of the cytokinin isopentenyladenine riboside (iPR) by hypocotyls was reduced more than $40 \%$ in ent3-1 [26]. However, information about physiological role of this transporter in terms of cytokinin function is limited to the data showing decreased sensitivity of root growth of the ent3-1 mutant to exogenous cytokinin ribosides [26]. Since ENT3 is highly expressed in the root vasculature of seedlings carrying the AtENT3-promoter-GUS construct [22], this carrier is likely to function in roots and to influence cytokinin export to the shoots. However, until now no indication of a physiological role of any ENT in the control of concentration of endogenous cytokinins in the roots and their distribution between roots and shoot has been provided.

The aim of the present study was to unravel a possible role of ENT3 in the uptake of cytokinins into root cells and its linkage with corresponding alterations in cytokinin distribution between roots and shoots and effects on root growth. To do so, the content and distribution of endogenous and exogenous cytokinins were quantified in the ent3-1 mutant and its wild type, Col. In addition, expression of cytokinin biosynthetic genes encoding isopentenyl transferase (IPT), activity of cytokinin oxidases, and root growth of ent3-1 mutant and its wild type, Col were studied. To unravel possible involvement of ENT in plant responses to availability of mineral nutrients, plants were either supplied 
with sufficient mineral nutrients or grown under nutrient limitation. We described for the first time the physiological role of ENT3 transporter in the regulation of cytokinin level (both the uptake and distribution) in shoots and roots and its effects on root elongation in Arabidopsis. The results suggest that ENT3 transporter distributes endogenous cytokinins between the apoplast and the symplast by facilitating cytokinin uptake by root cells, thereby preventing their long distance transport from roots to shoots.

\section{Materials and Methods}

\subsection{Plant Material and Growth Conditions}

The experiments were performed with Arabidopsis plants (Arabidopsis thaliana [L.] Heynh.) of wild type (WT) ecotype Columbia (Col) and ent3-1 (SALK_131585) mutant impaired in the function of ENT3 (AGI code; At4g05120) nucleoside transporter [22]. After stratification on wet filter paper in Petri dishes for 3 days at $4{ }^{\circ} \mathrm{C}$, the seeds were transferred to $100 \mathrm{~mL}$ vessels filled with sand saturated with 0.1 -strength Hoagland-Arnon solution and grown in an MLR-350H controlled-climate chamber (Sanyo, Osaka, Japan) for 28 days at a temperature of $23 / 19{ }^{\circ} \mathrm{C}$ (day/night), relative air humidity of $80 \%$, photosynthetically active radiation of $120 \mu \mathrm{mol} /\left(\mathrm{m}^{2} \mathrm{~s}\right) \mathrm{PAR}$, and 16-h-long photoperiod as described in [27]. A 0.1-strength of Hoagland-Arnon solution was chosen in preliminary experiments as optimal for maintaining Arabidopsis growth. Every day, the plants were supplied with $3 \mathrm{~mL}$ of Hoagland-Arnon solution and distilled water by the weight of vessels for the maintenance of sand hydration at $60 \%$ of full water capacity as described earlier [28]. Effects of the shortage of mineral nutrients on cytokinin content and root elongation was studied in experiments, where sand substrate was washed with $75 \mathrm{~mL}$ of 0.01 -strength Hoagland-Arnon solution taken in a volume five times greater than that of the solution in containers used for plant growing (4-week-old plants). To study the uptake of exogenous cytokinins by the plants, 3-week-old seedlings were placed into container with microplates with holes made in their bottom. Microplates were floating over the surface of aerated nutrient solution in 5-L-containers. One week after placing the plants into hydroponics, trans-zeatin riboside was added to the solution to yield final concentration of $4 \times 10^{-7} \mathrm{M}$.

Hormone sampling for cytokinins assay was performed 1 and 5 days after addition of zeatin riboside and sand washing, correspondingly. Activity of cytokinin oxidases and levels of the transcripts of isopentenyl transferase genes were measured in 4-week-old plants grown in the sand. Root length was estimated 7 days after sand washing.

\subsection{Cytokinin Extraction, Purification, and Immunoassay}

Shoots and roots of Arabidopsis plants were homogenized in $80 \%$ ethanol and kept overnight at $4{ }^{\circ} \mathrm{C}$. After filtration, all traces of ethanol were removed by vacuum evaporation and cytokinins from aqueous residue were purified as described [29,30]. In short, the hormones were concentrated on C18 column (Waters, Milford, MA, USA) and eluted with $5 \mathrm{~mL}$ of $80 \%$ ethanol. The dry residues dissolved in small volumes of ethanol were applied to TLC plates and were developed in a mixture of 2-butanol and $\mathrm{NH}_{4} \mathrm{OH}$ for separation of cytokinin bases and their derivatives. This procedure enabled separation and assay of cytokinin nucleotide ( $R f 0-0.1$ ), cytokinin glucosides ( $R f$ 0.1-0.2), and riboside of zeatin (ZR, Rf 0.4-0.5). Anti-trans-ZR rabbit serum was used for the enzyme-linked immunosorbent assay of cytokinins and its specificity has been described previously [2,31,32]. In short, enzyme immunoassay was performed with the protocol in which a conjugate of ZR to ovalbumin is absorbed onto the solid phase. A mixture of standard or sample plus specific serum was added to each well and incubated. Unbound rabbit antibodies were washed away and goat anti-rabbit IgG, conjugated to peroxidase, was incubated with the adsorbed antigen-antibody complex. All wells were again washed and the substrate solution consisting of o-phenylene-diamine was added. Color developed was quantitated at $492 \mathrm{~nm}$ with a microphotometer. Antibodies had high immunoreactivity towards corresponding zeatin and their derivatives (ribosides, 9-N-glucosides, and nucleotides) and low cross-reactivity 
to cis- $\mathrm{Z}$ and iP-type cytokinins (less than 1\%). This method has proven to be reliable by testing its results against physico-chemical assay $[28,29,33]$.

\subsection{Measurement of Cytokinin Oxidase Activity}

Activity of cytokinin oxidase was detected as described previously [4,34]. Leaves were homogenized in $0.1 \mathrm{M}$ imidazole buffer $(\mathrm{pH} 7.1$ ) and centrifuged at $12,000 \times g$ for $30 \mathrm{~min}$. To separate hormones from high molecular weight fraction, equal volume of saturated solution of ammonium sulfate was added to the supernatant and the pellet was resuspended in $0.1 \mathrm{M}$ imidazole buffer ( $\mathrm{pH}$ 7.1). Then, $30 \mathrm{ng}$ of cytokinin isopentenyl adenine (iP) added as a substrate to the suspension followed by incubation for $3 \mathrm{~h}$ at $37^{\circ} \mathrm{C}$. Cold ethanol was added to each sample to yield final $60 \%$ concentration $(v / v)$, the resulting suspension was kept at $4{ }^{\circ} \mathrm{C}$ for $40 \mathrm{~min}$ and then centrifuged at $5000 \times g$ for $10 \mathrm{~min}$. The decline in iP content in supernatant was detected with the help of immunoassay using antibodies against iPA. The absence of iP conversion to zeatin or dihydrozeatin was checked with immunoassay using corresponding antibodies. The presence of imidazole and $\mathrm{Cu}^{2+}$ accelerated the decay of iP confirming that the decline in iP level was due to its conversion to non-immunoreactive adenine catalyzed by cytokinin oxidase.

\subsection{Expression Analysis}

PCR with real-time product detection was performed as described previously [35]. RNA was isolated from the roots and leaves of plants of original ecotype and ent3-1 mutant with Trizol. Total RNA was treated with RQ1 DNAase free of RNAases (Promega; http:/ / www.promega.com/ (accessed on 18 December 2020)), then reverse transcription was performed using oligo (dT) primer and M-MuLV-reverse transcriptase (NEB New England Biolabs, Ipswich, MA, USA). In order to quantify transcripts of the genes encoding isopentenyl transferase (AtIPT1, AtIPT3, AtIPT5, and AtIPT7), we used primers described earlier [12]. Realtime RT-PCR was conducted in a Rotor-GeneTM 6000 thermocycler (Corbett Research, Mortlake, NSW, Australia) in the presence of intercalator SYBR Green. As an internal standard, we used a household gene of Arabidopsis AtACT2 (At3g18780). Calculations were made using the 2(-Delta C(T)) method [36], and the mRNA level of the elongation factor AtACT was defined as $100 \%$.

\subsection{Statistics}

The experiments were repeated three times with 3-20 replicates. Data were expressed as means $\pm \mathrm{SE}$, which were calculated in all treatments using MS Excel. A significant (at $p<0.05)$ difference between the means was calculated using $t$-test

\section{Results}

3.1. Concentration of Cytokinins and Their Metabolism (Cytokinin Oxidase Activity and Expression of AtIPT Genes) in ent3-1 Mutant and Col Plants

Content of cytokinins in the roots of hydroponically grown mutant plants (ent3-1) was lower than in WT (Col) plants (Figure 1a). This regularity has also been detected in plants grown in the sand. Activity of cytokinin oxidase either in shoots or roots was the same in the mutant and WT plants and consequently the decreased level of cytokinins detected in the roots of ent3-1 plants could not be due to the activity of cytokinin degradation in the plants (Figure 1b). 

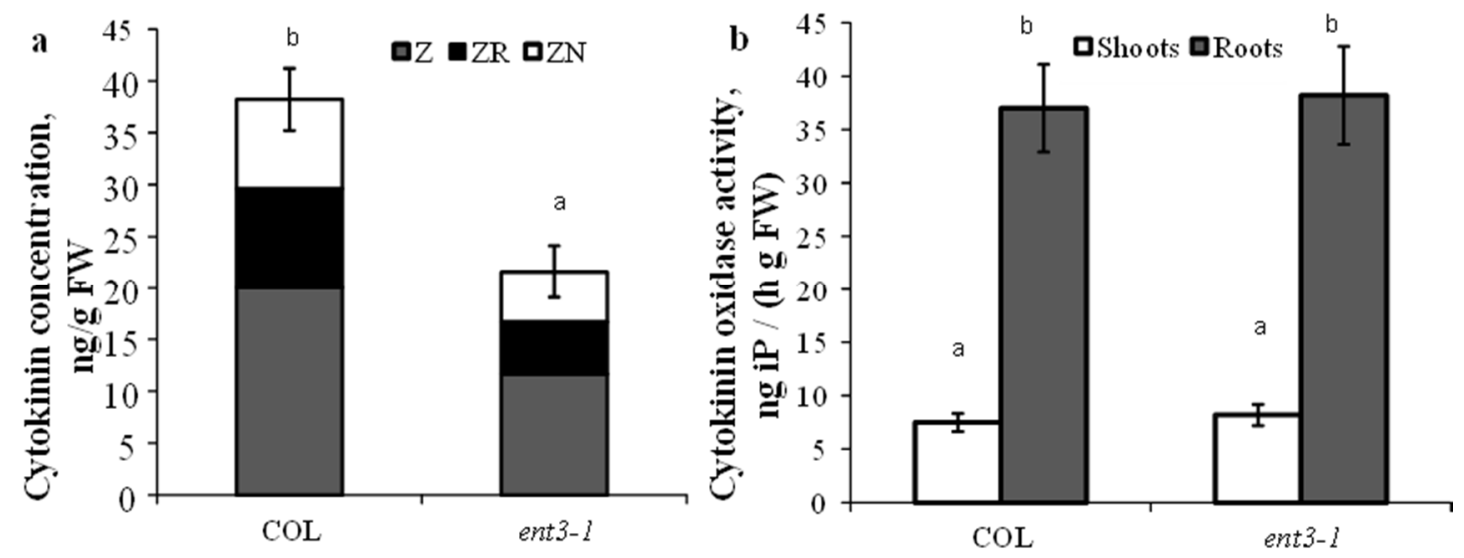

Figure 1. Concentration of zeatin (Z), zeatin riboside (ZR), and zeatin nucleotide (ZN) in roots (a) and cytokinin oxidase activity (b) in hydroponically grown 4-week-old ent3-1 Arabidopsis mutant and its parent ecotype Columbia (Col). Statistically differing means $(\mathrm{n}=6)$ are indicated with different letters $(p<0.05$, Student's $t$-test).

RT-PCR analysis of the transcripts of IPT-genes known to be expressed in the roots [12] showed that expression of the genes was not lower in ent3-1 than in Col thereby excluding possibility of attributing the decreased level of cytokinins in the roots of the mutant to their reduced synthesis (Figure 2).

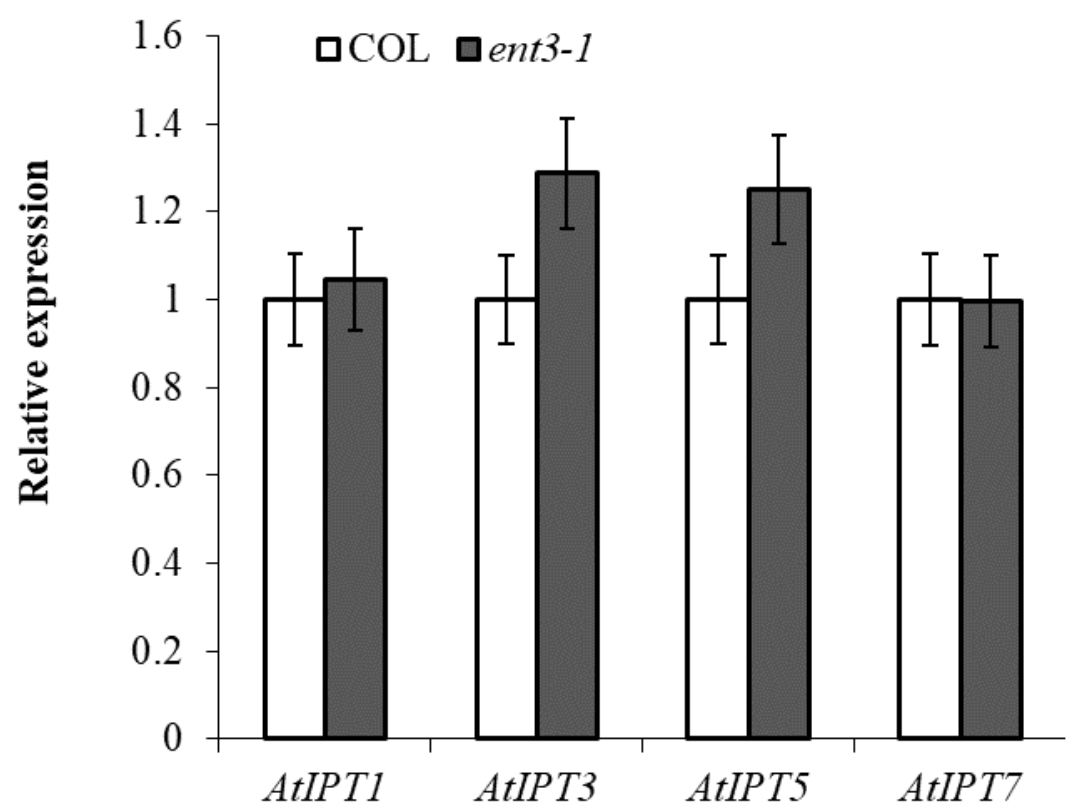

Figure 2. Level of transcripts of IPT1 (AT1G68460.1), IPT3 (AT3G63110.1), IPT5 (AT5G19040.1), and IPT7 (AT3G23630.1) in the roots of 4-week-old ent3-1 mutant and its parent ecotype Columbia (Col). Average expression levels from three replicates were normalized to actin with wild type scaled to $1(\mathrm{n}=3)$.

\subsection{Concentration of Cytokinins in Plants Treated with Zeatin Riboside}

In plants grown hydroponically in the solution without zeatin riboside, root concentration of cytokinins was higher in WT (Col) than in the mutant (ent3-1) (Figure 3a). Although addition of zeatin riboside to the nutrient solution increased concentration of cytokinins in plants of both genotypes, concentration of these hormones was still higher in the roots of Col than ent3-1. The opposite pattern was detected in the shoots (Figure 3b)—addition of zeatin riboside resulted in greater increase in cytokinin concentration in shoots of the mutant than in WT. Furthermore, plant treatment with zeatin riboside most significantly influenced the content of zeatin riboside in shoots, whose percentage out of the total con- 
tent of measured cytokinins increased from $23 \%$ in the control of both genotypes (plants untreated with exogenous cytokinins) to $50 \%$ in cytokinin-treated $\mathrm{Col}$ and to $65 \%$-in ent $3-1$.
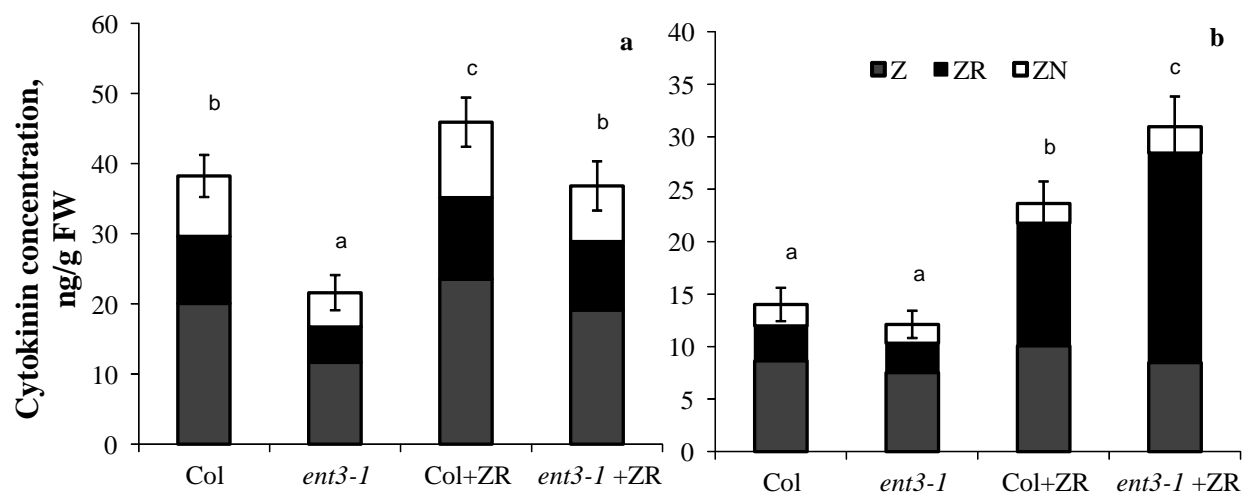

Figure 3. Concentration of zeatin $(\mathrm{Z})$, zeatin riboside $(\mathrm{ZR})$, and zeatin nucleotide $(\mathrm{ZN})$ in roots (a) and shoots (b) of hydroponically grown 4-week-old ent3-1 Arabidopsis mutant and its parent ecotype Columbia (Col) 1 day after zeatin riboside application $(\mathrm{Col}+\mathrm{ZR}$, ent3-1+ZR) to 0.1 Hoagland-Arnon nutrient solution (to yield $\left.4 \times 10^{-7} \mathrm{M}\right)$. Statistically differing means $(n=6)$ are indicated with different letters $(p<0.05$, Students $t$-test).

\subsection{Effects of Dilution of the Nutrient Solution on Cytokinin Concentration}

Dilution of the nutrient solution resulted in a decline in cytokinin content in the roots of both genotypes (Figure 4a) grown in sand. The percentage of zeatin riboside decreased from 30 to $20 \%$ out of the total sum of measured cytokinins in the plants of both genotypes.
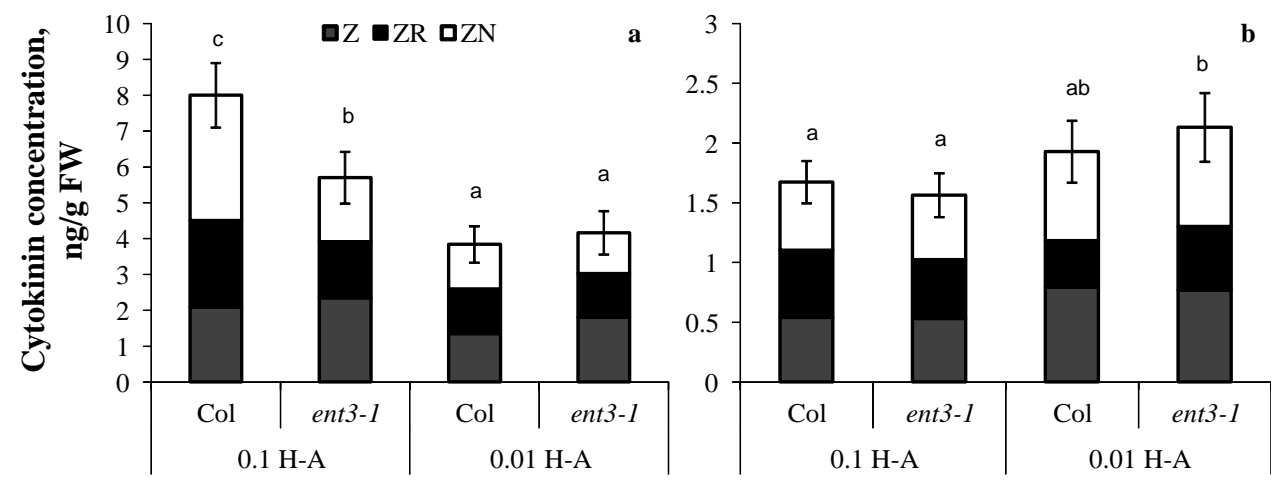

Figure 4. Concentration of zeatin (Z), zeatin riboside (ZR), and zeatin nucleotide (ZN) in roots (a) and shoots (b) of 5-weekold ent3-1 Arabidopsis mutant and its parent ecotype Columbia (Col) grown in vessels filled with sand saturated with 0.1-strength Hoagland-Arnon solution and sampled 5 days after dilution $(0.01 \mathrm{H}-\mathrm{A})$ of optimal 0.1 strength Hoagland-Arnon nutrient solution $(0.1 \mathrm{H}-\mathrm{A})$. Statistically differing means $(\mathrm{n}=6)$ are indicated with different letters $(p<0.05$, Students $t$-test).

The decline in concentration of mineral nutrients was accompanied by activation of root elongation-in $\mathrm{Col}$ the roots were $22 \%$ longer than in the control (Col plants supplied with sufficient mineral nutrition) and in ent3-1 roots were $14 \%$ longer than in the corresponding control (Figure 5). Col roots were shorter than those of ent3-1 under both levels of mineral nutrition (Figure 5). 


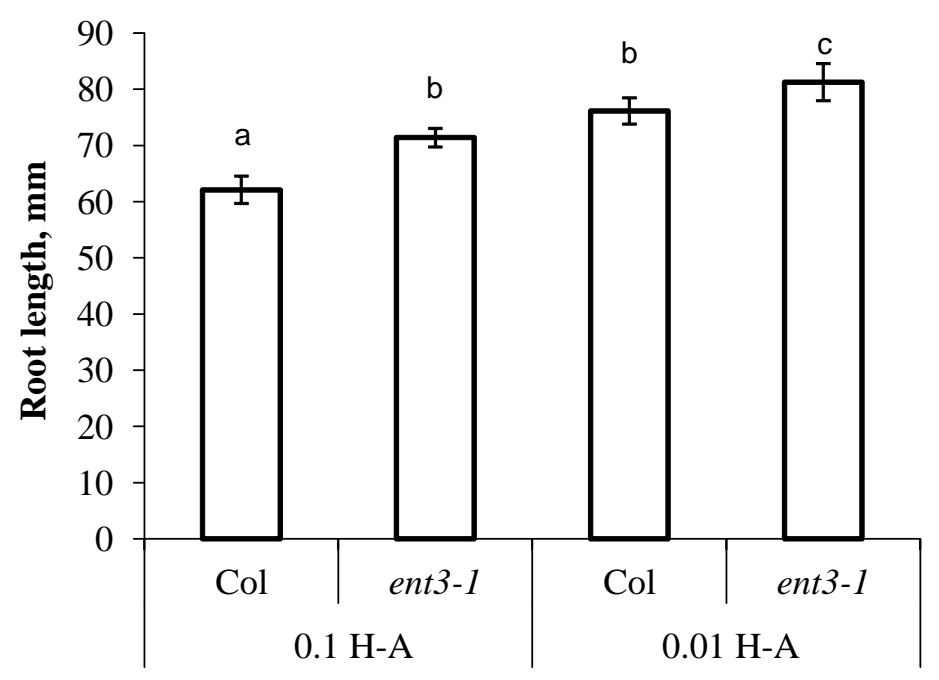

Figure 5. The length of the primary root of 5 weeks old ent3-1 Arabidopsis mutant and its parent ecotype Columbia (Col) 7 day after dilution (0.01 H-A) of optimal 0.1 strength Hoagland-Arnon nutrient solution $(0.1 \mathrm{H}-\mathrm{A})$. Statistically differing means $(\mathrm{n}=20)$ are indicated with different letters $(p<0.05$, Students $t$-test).

\section{Discussion}

ENT was characterized as a cellular importer of nucleosides including cytokinin ribosides $[25,26]$. Involvement of ENT in cytokinin transport was demonstrated in experiments with yeast cells expressing rice OsENT2 [37]. Meanwhile, information about its involvement in the control of cytokinin distribution in plants as well as in the implementation of the growth-regulating function of cytokinins is still scarce. Before our experiments were performed, this information was limited to the data showing decreased sensitivity of the root growth to exogenous cytokinin ribosides in the ent3-1 mutant [26]. The in vivo ENT3 function was supported by the study of double knockout plants in which both ENT3 and the extracellular nucleoside hydrolase NSH3 genes (ENT3::NSH3) were inactivated-these plants accumulated adenosine and uridine in the apoplast of leaves [38]. As ENT3 is highly expressed in the root vasculature of seedlings carrying AtENT3-promoter-GUS construct, a similar function in roots is highly likely [22]. Roots are a site for the synthesis of cytokinin ribosides which are transport form of cytokinins and precursors of active cytokinin bases [39]). Accordingly, ENT3 might function in cellular uptake of cytokinin ribosides, their conversion or signaling purposes in roots, thereby prohibiting export to the shoot via the xylem. This assumption is supported by the present experiments. A need for such uptake systems was proposed before [40] and a responsiveness of ENTs (ENT3 and ENT6) towards cytokinin treatment was shown [40]. However, until now no indication of a physiological role of any ENT in cytokinin metabolism was given. Reduced cytokinin riboside levels in roots of ent3-1 mutants without or after ZR treatment shown here, fits nicely to a proposed function of ENT3 as cellular ZR importer in roots (Figure 3). Altered cytokinin synthesis or degradation as a reason for the observation of reduced cytokinin riboside levels in roots of the ent3-1 mutant were ruled out as corresponding activities of CKX and expression of IPT genes were unchanged by the mutation.

Experiments with the uptake of exogenous zeatin riboside revealed that the decreased capacity of the ent3-1 mutant for accumulation of cytokinins in roots contributes to increased export of these hormones to the shoots. This was suggested by the lower level of overall accumulation of cytokinins in the roots and the increased level of these hormones in shoots of the ent3-1 mutant treated with exogenous zeatin riboside (as compared to Col). Another evidence of higher delivery of zeatin riboside to the shoots of the mutant is in greater increase in the percentage of this form of cytokinins in the shoots of the mutant treated with exogenous zeatin riboside. It was increased up to $65 \%$ out of total measured cytokinin content in the ent3-1 mutant, while zeatin riboside reached only a $50 \%$ level in 
Col. Sun et al. [26] detected reduced uptake of iPR and ZR by the mutant. Nevertheless, in those experiments uptake of ribosides by the mutant was measured in hypocotyls and changes in distribution of cytokinins between roots and shoots have been observed for the first time in the present work.

It has been shown by us previously that the outflow of exogenous zeatin to the shoots of wheat plants is increased as a result of protonophore-dependent inhibition of secondary active uptake of cytokinins by root cells resulting in higher cytokinin concentration in the apoplast of the xylem vessels [29]. Present experiments suggest that uptake of cytokinins by root cells enables retention of cytokinins in the root cells of the vasculature, where ENT3 has been shown to be located in the previous experiments [26]. This process is likely to prevent cytokinin export to shoots through the xylem. Although in the previous experiments we were likely to inhibit PUP transporters, Arabidopsis ENT works in a similar way, i.e., as riboside-proton cotransporters. In accordance, we can interpret the present results as suggesting that knocking out the gene coding for the transporter of ribosides decreases capacity of root cells to retain cytokinins in the root cells contributing to their apoplast loading into xylem and transport to the shoots through xylem. The choice of trans-zeatin for supplying roots with exogenous cytokinin in our experiments is supported by information that trans-zeatin-type species are the major form in xylem sap [39], while we used antibodies raised against trans-zeatin riboside having low cross-reactivity to cis-zeatin forms.

This hypothesized mechanism is illustrated in Figure 6. The importance of the apoplast pathway with a transpiration stream for the delivery of zeatin riboside to the shoots has been demonstrated in experiments with the uptake of this hormone by wheat plants showing its decline in the plants under decreased transpiration, i.e., under conditions limiting apoplast pathways [41]. The effect of ENT on cytokinin transport to the shoots suggested by the present experiments is likely to be opposite to that of ABCG14 transporters. While the latter contributes to xylem loading of cytokinins and thereby enables their transport to shoots and the decline in cytokinins accumulation in roots [14], ENT3, on the contrary, is likely to decrease cytokinins delivery to shoots due to the ENT-driven uptake of these hormones by root cells bringing about the retention of cytokinins in roots.

The response to the deficit in mineral nutrients was similar in the plants of both genotypes. We detected a decline in root cytokinins of either ent3-1 or Col accompanied by acceleration of root elongation. Similarity in the responses of the mutant and WT plants to dilution of the nutrient solution suggests that the membrane transport of cytokinin ribosides does not play a significant role in the decline in root cytokinins level under the shortage of mineral nutrients. An indirect evidence of this is in a reduced percentage of zeatin riboside registered by us in the shoots of both genotypes (from 30 to $20 \%$ out of the sum of all measured cytokinins). Our results are in accordance with those of Sun and co-authors [26], who detected an increased sensitivity to exogenous cytokinin riboside under nitrogen deficit that may serve as indirect evidence of decreased level of this form of cytokinins in nitrate-deficient plants.

Roots of the ent3-1 mutant were longer than those of Col, while nutrient deficiency activated their elongation. This response is in accordance with the initial level of cytokinins that was lower in the mutant than in WT as well as with the decline in the hormone level detected in the nutrient-deficient plants. This correspondence is explained by the inhibitory effect of cytokinins on root elongation [9] (longer roots were characteristic of the plants with decreased level of cytokinins [7]). The results are in accordance with those of Sun et al., who detected inhibition of root growth in the plants treated with cytokinin riboside [26] and decreased inhibitory effect of exogenous cytokinins on the ent3-1 mutant. The latter regularity may be due to decreased capacity of the ent3-1 mutant to accumulate cytokinins in roots revealed in our experiments bringing about the reduced inhibitory effect of cytokinin ribosides on the root growth. 


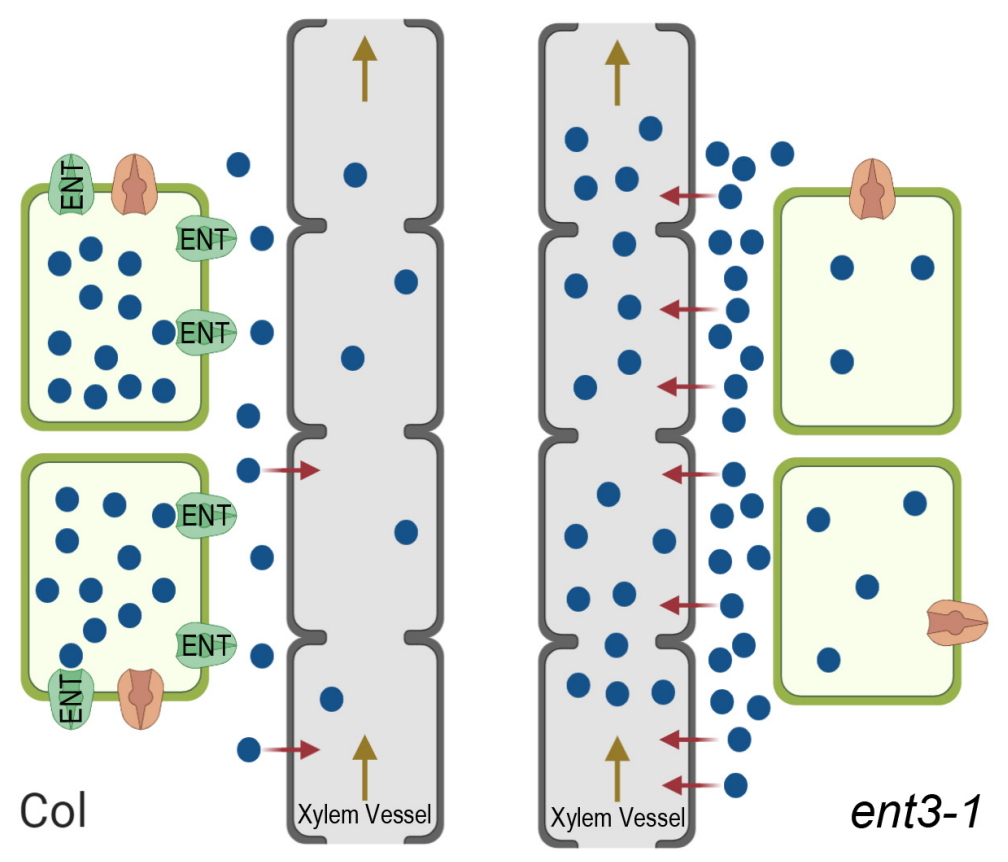

Figure 6. Scheme showing ENT-mediated uptake of externally supplied cytokinin riboside (blue circles) by living root cells, their passive loading into the dead xylem vessels (red arrows), and their flow to the shoot within the transpiration stream (brown arrow) in wild type Col plants (left) and ent3-1 mutant (right). In Col, uptake and retention of cytokinin ribosides by living root cells occurs at a high rate resulting in a decreased apoplastic concentration, low loading into xylem vessels through the apoplast route, and reduced export to the shoots with the transpiration stream. In the ent3-1 mutant cellular uptake of cytokinin riboside is reduced resulting in increased apoplast concentration, higher loading into the xylem apoplast, and greater export to shoots. Equivalent effects on endogenous cytokinins would indicate that the fully functional ENT3 carrier helps suppress losses to the shoot thereby maintaining the cytokinin titer of growing root cells at optimal levels.

Biochemical peculiarities have been previously studied in nitrate-deficient ent3-1 plants [22]. As far as we know, growth responses of the mutant to the deficiency in mineral nutrients had not been studied before our experiments were performed. Although the growth response was found to be similar in the plants of both genotypes, longer roots of the ent3-1 mutant than of WT plants detected either under normal mineral nutrition or its deficit suggest a significant role of the ENT3 transporter in the control of cytokinin levels in the roots and the rate of their elongation.

\section{Conclusions}

Thus, comparison of cytokinin concentration and distribution between shoots and roots of WT and plants with the mutated gene coding for ENT3 showed implication of this transporter in the regulation of cytokinin levels in the plants, thereby enabling cytokinin-dependent regulation of root elongation in Arabidopsis. The changes in cytokinin distribution between roots and shoots resulting from mutation of the ENT3 transporter gene have not been detected previously and were observed for the first time in the present work. The results suggest that uptake of cytokinins by root cells facilitated by ENT prevents their long-distance transport from roots to shoots, while a decline in the functioning of the transporter contributes to cytokinins export to the shoots. Control of cytokinins accumulation in the roots brought about by ENT transporters is likely to be involved in the regulation of root growth. ENT3 may also participate in modulating the balance of $\mathrm{CK}$ distribution between shoots and roots, which is important for the coordination of development of both parts of plants. 


\begin{abstract}
Author Contributions: Conceptualization, G.K. and T.M.; methodology, B.K.; formal analysis, D.V.; investigation, A.K. and B.K.; data curation A.K.; writing-original draft preparation, G.K.; writingreview and editing, T.M.; funding acquisition, D.V. All authors have read and agreed to the published version of the manuscript.
\end{abstract}

Funding: This research was partially supported by Russian Foundation for Basic Research No. 20-04-00305.

Conflicts of Interest: The authors declare no conflict of interest.

\title{
References
}

1. Havlová, M.; Dobrev, P.I.; Motyka, V.; Storchová, H.; Libus, J.; Dobrá, J.; Malbeck, J.; Gaudinová, H.; Vanková, R. The role of cytokinins in responses to water deficit in tobacco plants over-expressing trans-zeatin O-glucosyltransferase gene under 35S or SAG12 promoters. Plant Cell Environ. 2008, 31, 341-353. [CrossRef] [PubMed]

2. Kudoyarova, G.R.; Vysotskaya, L.B.; Cherkozyanova, A.; Dodd, I.C. Effect of partial rootzone drying on the concentration of zeatin type cytokinins in tomato (Solanum lycopersicum L.) xylem sap and leaves. J. Exp. Bot. 2007, 58, 161-168. [CrossRef] [PubMed]

3. Kiba, T.; Kudo, T.; Kojima, M.; Sakakibara, H. Hormonal control of nitrogen acquisition: Roles of auxin, abscisic acid, and cytokinin. J. Exp. Bot. 2011, 62, 1399-1409. [CrossRef]

4. Vysotskaya, L.B.; Korobova, A.V.; Veselov SYu Dodd, I.C.; Kudoyarova, G.R. ABA mediation of shoot cytokinin oxidase activity: Assessing its impacts on cytokinin status and biomass allocation of nutrient deprived durum wheat. Funct. Plant Biol. 2009, 36, 66-72. [CrossRef]

5. Vysotskaya, L.B.; Trekozova, A.W.; Kudoyarova, G.R. Effect of phosphorus starvation on hormone content and growth of barley plants. Acta Physiol. Plant. 2016, 38, 108. [CrossRef]

6. Wang, Q.; Zhu, Y.; Zou, X.; Li, F.; Zhang, J.; Kang, Z.; Li, X.; Yin, C.; Lin, Y. Nitrogen deficiency-induced decrease in cytokinins content promotes rice seminal root growth by promoting root meristem cell proliferation and cell elongation. Cells $2020,9,916$. [CrossRef] [PubMed]

7. Werner, T.; Motyka, V.; Laucou, V.; Smets, R.; van Onckelen, H.; Schmülling, T. Cytokinin-deficient transgenic Arabidopsis plants show multiple developmental alterations indicating opposite functions of cytokinins in the regulation of shoot and root meristem activity. Plant Cell 2003, 15, 2532-2550. [CrossRef]

8. Kudoyarova, G.R.; Dodd, I.C.; Veselov, D.S.; Rothwell, S.A.; Veselov, S.Y. Common and specific responses to availability of mineral nutrients and water. J. Exp. Bot. 2015, 66, 2133-2144. [CrossRef]

9. Ivanov, V.B.; Filin, A.N. Cytokinins regulate root growth through its action on meristematic cell proliferation but not on the transition to differentiation. Funct. Plant Biol. 2018, 45, 215-221. [CrossRef]

10. Korobova, A.V.; Vysotskaya, L.B.; Vasinskaya, A.N.; Kuluev, B.R.; Veselov, S.Y.; Kudoyarova, G.R. Dependence of root biomass accumulation on the content and metabolism of cytokinins in ethylene-insensitive plants. Russ. J. Plant Physiol. 2016, 63, 597-603. [CrossRef]

11. Takei, K.; Takahashi, T.; Sugiyama, T.; Yamaya, T.; Sakakibara, H. Multiple routes communicating nitrogen availability from roots to shoots: A signal transduction pathway mediated by cytokinin. J. Exp. Bot. 2002, 53, 971-977. [CrossRef]

12. Miyawaki, K.; Matsumoto-Kitano, M.; Kakimoto, T. Expression of cytokinin biosynthetic isopentenyltransferase genes in Arabidopsis: Tissue specificity and regulation by auxin, cytokinin, and nitrate. Plant J. 2004, 37, 128-138. [CrossRef]

13. Sakakibara, H.; Takei, K.; Hirose, N. Interactions between nitrogen and cytokinin in the regulation of metabolism and development. Trend Plant Sci. 2006, 11, 440-448. [CrossRef]

14. Zhang, K.; Novak, O.; Wei, Z.; Gou, M.; Zhang, X.; Yu, Y.; Yang, H.; Cai, Y.; Strnad, M.; Liu, C.-J. Arabidopsis ABCG14 protein controls the acropetal translocation of root-synthesized cytokinins. Nat. Commun. 2014, 5, 3274. [CrossRef]

15. Zhao, J.; Yu, N.; Fan, B.; Zhang, Y.; Zhu, E.; Zhang, M.; Zhang, K. ABC transporter OsABCG18 controls the shootward transport of cytokinins and grain yield in rice. J. Exp. Bot. 2019, 70, 6277-6291. [CrossRef]

16. Burkle, L.; Cedzich, A.; Dopke, C.; Stransky, H.; Okumoto, S.; Gillissen, B.; Kuhn, C.; Frommer, W.B. Transport of cytokinins mediated by purine transporters of the PUP family expressed in phloem, hydathodes, and pollen of Arabidopsis. Plant J. 2003, 34, 13-26. [CrossRef] [PubMed]

17. Möhlmann, T.; Mezher, Z.; Schwerdtfeger, G.; Neuhaus, H.E. Characterisation of a concentrative type of adenosine transporter from Arabidopsis thaliana (ENT1, At). FEBS Lett. 2001, 509, 370-374. [CrossRef]

18. Duran-Medina, I.; Diaz-Ramirez, D.; Marsch-Martinez, N. Cytokinins on the Move. Front. Plant Sci. 2017, 8, 146. [CrossRef]

19. Zürcher, E.; Liu, J.; di Donato, M.; Geisler, M.; Müller, B. Plant development regulated by cytokinin sinks. Science 2016, 353, 1027-1030. [CrossRef]

20. Zürcher, E.; Tavor-Deslex, D.; Lituiev, D.; Enkerli, K.; Tarr, P.T.; Müller, B. A robust and sensitive synthetic sensor to monitor the transcriptional output of the cytokinin signaling network in planta. Plant Physiol. 2013, 161, 1066-1075. [CrossRef]

21. Hirose, N.; Takei, K.; Kuroha, T.; Kamada-Nobusada, T.; Hayashi, H.; Sakakibara, H. Regulation of cytokinin biosynthesis, compartmentalization and translocation. J. Exp. Bot. 2008, 59, 75-83. [CrossRef] 
22. Cornelius, S.; Traub, M.; Bernard, C.; Salzig, C.; Lang, P.; Möhlmann, T. Nucleoside transport across the plasma membrane mediated by equilibrative nucleoside transporter 3 influences metabolism of Arabidopsis seedlings. Plant Biol. 2012, 14, 696-705. [CrossRef]

23. Jung, B.; Hoffmann Ch Möhlmann, T. Arabidopsis nucleoside hydrolases involved in intracellular and extracellular degradation of purines. Plant J. 2011, 65, 703-711. [CrossRef]

24. Wormit, A.; Traub, M.; Flörchinger, M.; Neuhaus, H.E.; Möhlmann, T. Characterization of three novel members of the Arabidopsis thaliana equilibrative nucleoside transporter (ENT) family. Biochem. J. 2004, 383, 19-26. [CrossRef]

25. Traub, M.; Florchinger, M.; Piecuch, J.; Kunz, H.-H.; Weise-Steinmetz, A.; Deitmer, J.W.; Neuhaus, H.E.; Möhlmann, T. The fluorouridine insensitive 1 (fur1) mutant is defective in equilibrative nucleoside transporter 3 (ENT3), and thus represents an important pyrimidine nucleoside uptake system in Arabidopsis thaliana. Plant J. 2007, 49, 855-864. [CrossRef] [PubMed]

26. Sun, J.; Hirose, N.; Wang, X.; Wen, P.; Xue, L.; Sakakibara, H.; Zuo, J. Arabidopsis SOI33/AtENT8 gene encodes a putative equilibrative nucleoside transporter that is involved in cytokinin transport in planta. J. Integr. Plant Biol. 2005, 47, 588-603. [CrossRef]

27. Shtratnikova, V.Y.; Kudryakova, N.V.; Kudoyarova, G.R.; Korobova, A.V.; Akhiyarova, G.R.; Danilova, M.N.; Kusnetsov, V.V.; Kulaeva, O.N. Effects of nitrate and ammonium on growth of Arabidopsis thaliana plants transformed with the ARR5::GUS construct and a role for cytokinins in suppression of disturbances induced by the presence of ammonium. Russ. J. Plant Physiol. 2015, 62, 741-752. [CrossRef]

28. Arkhipova, T.N.; Prinsen, E.; Veselov, S.Y. Martinenko, E.V.; Melentiev, A.I.; Kudoyarova, G.R. Cytokinin producing bacteria enhances plant growth in drying soil. Plant Soil 2007, 292, 305-315. [CrossRef]

29. Kudoyarova, G.R.; Korobova, A.V.; Akhiyarova, G.R.; Arkhipova, T.N.; Zaytsev, D.Y.; Prinsen, E.; Egutkin, N.L.; Medvedev, S.S.; Veselov, S.Y. Accumulation of cytokinins in roots and their export to the shoots of durum wheat plants treated with the protonophore carbonyl cyanide m-chlorophenylhydrazone (CCCP). J. Exp. Bot. 2014, 65, 2287-2294. [CrossRef]

30. Arkhipova, T.N.; Evseeva, N.V.; Tkachenko, O.V.; Burygin, G.L.; Vysotskaya, L.B.; Akhtyamova, Z.A.; Kudoyarova, G.R. Rhizobacteria inoculation effects on phytohormone status of potato microclones cultivated in vitro under osmotic stress. Biomolecules 2020, 10, 1231. [CrossRef]

31. Veselov, S.U.; Valcke, R.; Van Onckelen, H.; Kudoyarova, G. Cytokinin content and location in the leaves of the wild-type and transgenic tobacco plants. Russ. J. Plant Physiol. 1999, 46, 26-33.

32. Arkhipova, T.N.; Veselov, S.U.; Melentiev, A.I.; Martynenko, E.V.; Kudoyarova, G.R. Ability of bacterium Bacillus subtilis to produce cytokinins and to influence the growth and endogenous hormone content of lettuce plants. Plant Soil 2005, 272, 201-209. [CrossRef]

33. Veselov, S.Y.; Timergalina, L.N.; Akhiyarova, G.R.; Kudoyarova, G.R.; Korobova, A.V.; Ivanov, I.; Arkhipova, T.N.; Prinsen, E. Study of cytokinin transport from shoots to roots of wheat plants is informed by a novel method of differential localization of free cytokinin bases or their ribosylated forms by means of their specific fixation. Protoplasma 2018, 255, 1581-1594. [CrossRef] [PubMed]

34. Veselov, S.Y.; Simonyan, M.V. Immunoenzyme analysis of cytokinins as an assay for cytokinin oxidase activity. Russ. J. Plant Physiol. 2004, 5, 266-270. [CrossRef]

35. Kuluev, B.; Avalbaev, A.; Mikhaylova, E.; Nikonorov, Y.; Berezhneva, Z.; Chemeris, A. Expression profiles and hormonal regulation of tobacco expansin genes and their involvement in abiotic stress response. J. Plant Physiol. 2016, 206, 1-12. [CrossRef]

36. Livak, K.J.; Schmittgen, T.D. Analysis of relative gene expression data usingreal-time quantitative PCR and the 2(-Delta Delta $\mathrm{C}(\mathrm{T})$ ) method. Methods 2001, 25, 402-408. [CrossRef]

37. Hirose, N.; Makita, N.; Yamaya, T.; Sakakibara, H. Functional characterization and expression analysis of a gene, OsENT2, encoding an equilibrative nucleoside transporter in rice suggest a function in cytokinin transport. Plant Physiol. 2005, 138, 196-206. [CrossRef]

38. Daumann, M.; Fischer, M.; Niopek-Witz, S.; Girke, C.; Möhlmann, T. Apoplastic nucleoside accumulation in Arabidopsis leads to reduced photosynthetic performance and increased susceptibility against Botrytis cinerea. Front. Plant Sci. 2015, 6, 1158. [CrossRef]

39. Sakakibara, H. Cytokinin biosynthesis and transport for systemic nitrogen signaling. Plant J. 2020. [CrossRef]

40. Kang, J.; Lee, Y.; Sakakibara, H.; Martinoia, E. Cytokinin transporters: GO and STOP in signaling. Trends Plant Sci. 2017, 22, 455-461. [CrossRef]

41. Korobova, A.V.; Vasinskaya, A.N.; Akhiyarova, G.R.; Veselov, S.Y.; Kudoyarova, G.R.; Hartung, W. Dependence of cytokinin distribution in plants on their physical and chemical properties and transpiration rate. Russ. J. Plant Physiol. 2013, 60, 193-199. [CrossRef] 